\title{
25th Anniversary of the Centre for Rheumatic Diseases
}

\author{
Maciej Brzeski, Rajan Madhok
}

The 25th anniversary of the Centre for Rheumatic Diseases, Tlasggow, was celebrated at a meeting entitled 'Rheumatology 1990'. Organised by the current consultants, Dr Hilary Capell and Dr Roger Sturrock, it was held in the Royal College of Physicians and Surgeons of Glasgow on 22-23 March 1990. Most of the speakers and chairmen were past or current staff of the Centre; the presence of so many at this event was a tribute to the affection inspired by the Centre and its founders-notably, Professor Watson Buchanan. Although part of the university department of medicine at the Royal Infirmary of Glasgow, the Centre was established in the separate disused fever hospital at Baird Street. It was soon recognised locally by the care its patients received, and world wide by the breadth and quality of the research. As with all small communities it was proud of its survival and growth, and its increasing renown.

The first session was devoted to the immunogenetics of rheumatic diseases. Dr John Bell outlined the complexity of the HLA region at the molecular level, and discussed studies of other regions that might unravel the immunogenetics of rheumatoid arthritis (RA). The structure of HLA molecules relative to antigen presentation to $\mathrm{T}$ cells was reviewed. Detection of the similarity in amino acid sequences of the $\beta_{1}$ chain third hypervariable region of DR1, DR4, Dw4, and Dw14 highlighted the achievements possible with the new tools available. Professor Connor balanced the perspective of the molecular biologists by reconciling the achievements of the old and new genetics.

The role of non-HLA-DR genes in the immunogenetic heterogepeity of RA was introduced by Dr David Grennan. HLA class III genes were shown to modify HLA-DR, resulting in rheumatoid vasculitis and Felty's syndrome. Class I HLA genes were not forgotten. Professor Verna Wright reviewed the clinical studies that led to the concept of the seronegative spondyloarthropathies. Dr Sturrock reminded the audience that environmental triggers in ankylosing spondylitis were first postulated by Davis Collie in 1875. He also drew from his clinical experience and summarised data produced in his laboratory and by others for environmental triggers in the HLA-B27 arthropathies.

Professor Keith Whaley reported on factors that inhibit complement action in immune complex diseases. He described a hepatocyte glycoprotein which inhibits complement activation and reduces its solubility, as well as binding to the Fc end of IgG. The therapeutic potential of this finding was raised in the discussion by Dr Tony Boyle.

Professor David Lawson chaired the session on existing treatments - as a member of the Committee on Safety of Medicines he pointed out that non-steroidal anti-inflammatory drugs (NSAIDs) were the most common agents for which yellow cards were submitted. Professor Brooks highlighted the non-prostaglandin effects of NSAIDs and expressed concern about sustained prostaglandin inhibition achieved within the inflamed joint by the longer acting agents. Dr Tony Boyle reflected both on the early days of the Centre and on dollars created for the pharmaceutical industry by NSAIDs. The FDA decision that NSAID packaging should carry a warning about the estimated $2-4 \%$ risk of gastrointestinal ulceration per year was regretted by Dr Boyle because he considered the figure was too low. Dr Capell emphasised the practical and statistical problems associated with long term studies of second line agents in $\mathrm{RA}$, and stressed the considerable co-morbidity often seen in patients with RA.

Using RA as a model, Dr Jill Belch presented data to support inhibition of the lipoxygenase pathway as beneficial in chronic inflammation. Alteration of the substrate from arachidonic acid to eicosapentaenoic acid or dihomo- $\gamma$ linolenic acid, thereby altering the class of eicosanoid to less proinflammatory products, was a potential alternative treatment.

Professor R Maini represented the Kennedy Institute, which is also celebrating 25 years of rheumatology. Studies at the Kennedy showed that a monoclonal antibody to tumour necrosis factor could downregulate interleukin-1 in vitro. Therapeutic approaches following this and other insights into interactions between antigen presenting and $T$ cells were discussed.

Parallels between RA and haemophilic arthritis were drawn by Dr Madhok, who emphasised that approaches familiar to the rheumatologist could be successfully applied to the treatment and perhaps prevention of haemophilic arthritis.

Professor George Nuki, eyeing 'The outlook for osteoarthritics', found little cheer. Our ignorance of cartilage degeneration and the lack of reliable biochemical markers for disease process and progress hindered a rational search for treatment. He could foresee no intervention 
to slow the rise of total hip replacements in the United Kingdom, from 40000 now to 150000 yearly by 2030 AD. Nor did Dr Kris Jasani offer hope that cartilage might be protected clinically. Drug mediated reduction of cartilage damage in animal models was far removed from patients presenting with extensive cartilage loss. Dr Saklatvala's work has shown interleukin-1 to participate in cartilage damage, probably by an initial reduction of proteoglycan content. Because only $1 \%$ of interleukin-1 receptors are used biologically, receptor blockade as treatment would be impractical. Post receptor pathways remain unknown and thus inviolate.

The session on osteoporosis was more optimistic. As hip fractures may bankrupt Medicare in America, prophylaxis seems appropriate. Dr Ignac Fogelman identified bone density as the best predictor of subsequent fracture. New scanners take only six minutes to identify perimenopausal women likely to benefit from intervention. Dr Stuart Ralston explained why oestrogen is currently the best prophylaxis. To increase bone density in established osteoporosis was more difficult, but etidronate seemed effective and had reduced the incidence of fracture.

Professor David Hamblen reviewed the increasingly successful collaboration between orthopaedic surgeon and bioengineer. Production of bespoke biomaterials to mimic wear particles might identify the cause of some of the damage implicated in prosthetic loosening. Yet however carefully a prosthetic joint is computer modelled, the success of implantation was most dependent on the surgeon's skill and artistry. The 'tiger territory' of high cervical spine surgery for rheumatoid disease was stalked by Mr Robin Johnson, neurosurgeon, and Mr Ian Kelly. Computed tomography and magnetic resonance imaging have improved assessment of cord compression, allowing surgery to be tailored to the patient's specific problem.

Reviewing a vast range of hand surgery, $\mathrm{Mr}$ Bob Simpson stressed two points: (a) restoration of function is more difficult than correction of deformity; (b) the earlier a hand surgeon is consulted, the more there is to offer. As an aside, many will be interested in the Weltanschaung of the hand specialist-'housework is made up of activities dangerous to the hands and should be avoided'. This is one paper our spouses will not be allowed to see.

'Dipping one foot in the grave' (the clinic of the paleopathologist), professor Paul Dieppe explained that the antiquity of rheumatoid arthritis had yet to be resolved, although Swedish neolithic and early British skeletons and pre-Columbian sites in Alabama hold bones which seem to suggest erosive disease. The audience was offered hands on experience of an ankylosed spine and an ancient femur. The grand finale was Professor Watson Buchanan's wide ranging review of how the past might guide the future. In contrast with the structured research previously described, he stressed the serendipity of some of the great therapeutic discoveries. William Heberden the Elder was cited with approval for not following the herd with fashionable but questionable treatment. Sitting in the audience, one felt drawn to compare the historical physician with the Titan before us.

The separate site at Baird Street Hospital was relinquished in 1984 when the Centre for Rheumatic Diseases moved into the 'mother ship' of the Royal Infirmary, and thus nearer the brewery and necropolis. Baird Street Hospital stands no more, but the spirit persists in the Centre and in the life of those who worked there. To the city motto 'Let Glasgow flourish' we would add 'Let Glasgow rheumatology flourish'. 